Background/introduction The duty doctor role was introduced into our integrated sexual health service in 2012 with the aim of improving patient flow through the clinic.

Aim(s)/objectives Three years on we wished to review the service and ensure it remains fit for purpose.

Methods We undertook an anonymous survey of clinical staff assessing if the duty doctor improved the service for patients and staff waiting for a second opinion; for staff teaching and learning and staff's confidence in duty doctor knowledge.

Results Twenty seven members of staff completed the questionnaire. When asked to rate the utility of the duty doctor on a scale of 1 to 10 (1-no use at all, 10-indispensable) the average response was 8 . The majority thought the service was better or much better for patients and staff. Only 21\% thought the service should be expanded. $72 \%$ of respondents have/would bypass duty doctor. 55\% would approach the duty doctor for GUM but ask elsewhere for contraception. $47 \%$ would approach for contraception but ask elsewhere for GUM. Only 25\% thought a separate contraception and GUM duty doctor was needed. The service was praised for its expert $2^{\text {nd }}$ opinions, quick responses and the reassurance to patients. Common problems were that the doctors were hard to contact at times and occasionally doctor's gender was difficult for patients.

Discussion/conclusion The duty doctor is a valuable role, accessibility needs to be addressed but with the exception of increasing the pool of doctors who act as duty doctor the role should remain unchanged.

\section{P177 DEVELOPING THE SEXUAL HEALTH WORKFORCE: DESIGNING AND DELIVERING TRAINING FOR HEALTHCARE ASSISTANTS}

Eleanor Lock, Michael Brady*. Kings College Hospital NHS Foundation Trust, London, UK

\subsection{6/sextrans-2015-052126.220}

Background Increasingly 'simple' sexual health services (e.g. asymptomatic screening) are provided by Healthcare Assistants (HCA's). There is no nationally accredited training for this staff group and clinical services usually provide in house training to develop their theoretical knowledge and skills.

Aim To develop and evaluate a course for HCA's working in sexual health.

Methods We designed a 2-day course covering 14 topics based on the structure of the STIF course. Content and learning objectives were devised using existing competencies and in consultation with the multi-disciplinary team. Nurses deliver the course using a mixed teaching methodology (lectures, role play and interactive workshops). Learning outcomes include:

Knowledge:

1. Understand the principles of asymptomatic STI testing

2. Understand issues relating to confidentiality, vulnerable patients and partner notification

Skills:

1. Feeling comfortable and competent taking a sexual history

2. Optimise care pathways with local relevant support services

Attitudes:

1. Understand the range of human sexualities, lifestyles and culture and their impact on transmission, prevention and counselling
Results The course has run on 2 occasions with a total of 18 attendees: both sexual health HCAs and practitioners from other specialities (e.g. A+E and gynaecology). All topics were well evaluated with a mean overall score of $4.55 / 5$ (range 3.8-5).

Free text comments were positive with specific reference to how "valuable", "useful" and "relevant" the course was.

Discussion We have designed, delivered and evaluated a successful sexual health course for HCAs that could easily be nationally accredited and delivered in other services and settings.

\section{P178 SPECIALIST HERPES CLINICS: IS THERE ANY POINT?}

${ }^{1}$ Sarah Wade*, ${ }^{1,2}$ Emily Clarke, ${ }^{1,2}$ Raj Patel. 'University of Southampton, Hampshire, UK; ${ }^{2}$ Royal South Hants Hospital, Southampton, UK

\subsection{6/sextrans-2015-052126.221}

Background/introduction Specialist clinics have been introduced in a number of specialities, but patient benefits have proved difficult to demonstrate, despite increased investigations and costs. Genital herpes requires extensive counselling which previous studies have demonstrated is often of poor quality.

$\operatorname{Aim}(\mathrm{s}) /$ objectives To assess the value of a specialist genital herpes clinic, in terms of patient satisfaction and outcomes, in comparison with a general genitourinary medicine clinic at a UK level 3 sexual health service in patients with a diagnosis of first episode genital herpes.

Methods 200 patient records of those attending a UK level 3 sexual health service with first episode herpes between 2012-2013 attending a specialist or general clinic were reviewed to assess initial management, complicating factors, and subsequent health seeking behaviour. 20 patients with a recent diagnosis of herpes attending either a specialist or general clinic were interviewed to determine patient satisfaction, and information provided on a number of key counselling topics identified by the BASHH herpes guidelines.

Results Provisional results from 79 patients demonstrate that those attending the specialist clinic were more likely to have complicating factors, including pregnancy, and psychological distress. Return to clinic with recurrences was $20 \%$ for the specialist and $15 \%$ for the general clinic. Full results will be available by the conference.

Discussion/conclusion Patients attending a specialist herpes clinic presented with more complicating factors, but despite this there was little difference between patient outcomes and satisfaction between clinics. Specialist herpes clinics may therefore be useful to manage more complex patients.

\section{P179 EVALUATION OF AN ONLINE BOOKING SERVICE TO ACCESS ASYMPTOMATIC SCREENING}

Anatole Menon-Johansson*, Ruslan Artykov. Guy's \& St Thomas' NHS Foundation Trust, London, UK

\subsection{6/sextrans-2015-052126.222}

Background/introduction Symptomatic patients are prepared to wait hours for open access sexual health services; however, patients without symptoms want simple and convenient access to testing.

Aim(s)/objectives We therefore introduced a new online booking service for asymptomatic patients and evaluated if patients would use it correctly. 